\title{
AVALIAÇÃO DAS PROPRIEDADES FÍSICAS E MECÂNICAS DE PAINÉIS DE FIBRAS DE MÉDIA DENSIDADE (MDF) PRODUZIDOS PELAS INDÚSTRIAS BRASILEIRAS
}

\author{
Luciane Paes Torquato ${ }^{1}$, Setsuo Iwakiri ${ }^{2}$, Ghislaine Miranda Bonduelle ${ }^{3}$, \\ Carlos Eduardo Camargo de Albuquerque ${ }^{2}$, Jorge Luís Monteiro de Matos ${ }^{2}$ \\ ${ }^{1}$ Eng $^{\mathrm{a}}$. Industrial Madeireira, M.Sc., UFPR, Curitiba, PR - 1_torquato@yahoo.com \\ ${ }^{2}$ Eng. Florestal, Dr., Depto. de Engenharia e Tecnologia Florestal, UFPR, Curitiba, PR - setsuo@ufpr.br - \\ camargo@ufpr.br - jmatos@ufpr.br \\ ${ }^{3} \mathrm{Eng}^{\mathrm{a}}$. Florestal, Dr ${ }^{\mathrm{a}}$., Depto. de Engenharia e Tecnologia Florestal, UFPR, Curitiba, PR - ghislaine@ufpr.br
}

Recebido para publicação: 13/05/2009 - Aceito para publicação: 31/08/2009

\begin{abstract}
Resumo
Este trabalho foi desenvolvido com o objetivo de avaliar a qualidade de painéis MDF produzidos pelas indústrias brasileiras com base nos resultados de propriedades físicas e mecânicas dos painéis tipo "standard" com $15 \mathrm{~mm}$ de espessura. Foram selecionadas quatro empresas produtoras de painéis MDF, que foram identificadas pelas letras A, B, C e D. Para as avaliações das propriedades dos painéis, foram, coletadas para todas as empresas, três painéis-amostras produzidos com madeira de pinus, além de painéis de eucalipto para a empresa $\mathrm{D}$. As propriedades avaliadas foram: teor de umidade, densidade, perfil de densidade, absorção de água e inchamento em espessura 2 e 24 horas, ligação interna e flexão estática. Os ensaios foram realizados de acordo com a norma europeia EM, e os resultados foram comparados com os requisitos da norma EN 622-5:2006. Os painéis produzidos com madeira de eucalipto apresentaram valores médios inferiores de ligação interna e média de absorção de água maior que os obtidos com painéis de pinus. Os valores médios de inchamento em espessura 24 horas, MOE e MOR em flexão estática paralela dos painéis MDF comerciais avaliados nesta pesquisa atendem aos requisitos mínimos da norma EN 622-5:2006. Os valores médios de ligação interna foram inferiores em relação aos requisitos dessa norma.

Palavras-chave: Painéis MDF; pinus; fibras de madeira.
\end{abstract}

\begin{abstract}
Evaluation of physical and mechanical properties of medium density fiberboard (MDF) manufactured from brazilian industries. This research was developed to evaluate the quality of MDF manufactured by brazilian industries based on the results of physical and mechanical properties of the standard boards with $15 \mathrm{~mm}$ thickness. Four factories were selected and identified as A, B, C, and D. For the evaluation of the board properties, they were collected from the all factories three samples produced by pine wood, besides of sample produced by eucalipto wood to the factory $\mathrm{D}$. The following board properties were evaluated: moisture content, density, density profile, water absorption and thickness swelling 2 and 24 hours, internal bond and static bending. The tests were carried out according to European Standard EN and the results were compared with the requirements of European Standard EN 622-5:2006. The boards manufactured from eucalipto wood showed lower average values of internal bond and higher water absorption in comparison to boards manufactured from pine wood. The average values of thickness swelling after 24 hours water soaking, MOE and MOR parallel static bending of the commercial MDF evaluated in this study attends to minimum requirements of EN 6225:2006. The average values of internal bond were lower than in relation to the EN standard.

Keywords: MDF; pine; wood fiber.
\end{abstract}

\section{INTRODUÇÃO}

A produção brasileira de painéis de madeira em 2005 foi de 7,7 milhões de $\mathrm{m}^{3} \mathrm{e}$ vem crescendo a uma taxa anual de 9,5\% desde 1995. Essa evolução pode ser atribuída a um conjunto de fatores, como a busca de alternativas à madeira maciça; a modernização tecnológica do parque fabril, que proporcionou a 
oferta de novos produtos, como MDF (médium density fiberboard) e OSB (oriented strand board), e a melhoria da qualidade tecnológica do aglomerado, com nova denominação comercial de MDP (médium density particleboard); e a redução dos juros e melhoria da renda, que deram fortes impulsos aos setores de construção civil e indústria moveleira, ambos consumidores de painéis de madeira (BNDES, 2008).

O contexto retratado acima está sendo comprovado no Brasil, principalmente em relação ao painel MDF, que está caracterizando uma forte expansão do mercado e em grandes volumes. Somente em 2007, as cinco empresas produtoras no país foram responsáveis pela fabricação de aproximadamente 1,88 milhões de $\mathrm{m}^{3}$ desse produto. Esses mesmos fabricantes estão ampliando suas unidades de produção e contarão com novos concorrentes no mercado a partir de 2008 e 2009. São seis novas empresas confirmadas que estão implantando ou com projetos de novas instalações industriais de MDF e há indícios de uma sétima empresa, que irá produzir o painel no norte do Brasil (BNDES, 2008).

A evolução do mercado para um painel de alto valor agregado, fabricado por empresas de grande porte e em grandes volumes, chamou a atenção para o fato de que, provavelmente, esses produtos serão disponibilizados no mercado com especificações iguais, respeitando as normas vigentes, com preços similares, mas com origem em processos produtivos com parâmetros diferentes e desconhecidos do consumidor final. Essa situação pode ter como consequência painéis MDF com características físicas e mecânicas bastantes diferenciadas e ofertados no mercado como o mesmo produto. Analisando essa evolução mercadológica, chegou-se à conclusão de que será essencial para as indústrias de painéis MDF estar acompanhadas de estudos e inovações para que esse produto e seus fabricantes permaneçam no mercado de forma competitiva (BNDES, 2008).

O MDF é definido como um painel produzido a partir de fibras de madeira aglutinadas com resina ureia-formaldeído (ou melamina-ureia-formaldeído) e consolidadas através de prensagem à alta temperatura e pressão, em densidades de $0,55 \mathrm{~g} / \mathrm{cm}^{3}$ (MDF light) a $0,85 \mathrm{~g} / \mathrm{cm}^{3}$ (HDF) (IWAKIRI, 2005). O painel MDF é produzido através do processo a seco, e aditivos químicos podem ser incorporados para melhorar algumas propriedades, como estabilidade dimensional, resistência ao fogo e agentes biodegradadores da madeira (MALONEY, 1993).

As indústrias brasileiras de painéis MDF adotam a norma europeia EN 622-5:2006 para avaliação das propriedades dos painéis utilizados em ambiente seco. Na tabela 1 estão apresentados os requisitos mínimos para as principais propriedades físico-mecânicas de painéis MDF, conforme estabelecido pela norma EN 622-5:2006.

Tabela 1. Requisitos da norma EN 622-5:2006.

Table 1. Requirements of EN 622-5:2006.

\begin{tabular}{lccc}
\hline Propriedade & $\begin{array}{c}\text { Método de } \\
\text { ensaios }\end{array}$ & Unidades & $\begin{array}{c}\text { Requisitos mínimos } \\
\text { (espessura 12 a 19 mm) }\end{array}$ \\
\hline Inchamento em espessura 24h & EN 317 & $(\%)$ & $>12$ \\
Ligação interna & EN 319 & $\mathrm{MPa}$ & $>0,55$ \\
Resistência a flexão (MOR) & EN 310 & $\mathrm{MPa}$ & $>20$ \\
Módulo de elasticidade (MOE) & EN 310 & $\mathrm{MPa}$ & $>2.200$ \\
\hline
\end{tabular}

O painel MDF é um produto que apresenta características muito similares à madeira sólida, tendo em vista ser um painel reconstituído de fibras individualizadas e aglutinadas por meio de ligações adesivas. É um produto com características superiores em comparação aos painéis de partículas aglomeradas, principalmente no que se refere à estabilidade dimensional e condições de usinagem, tanto nas bordas quanto nas faces. Com densidade adequada e perfeita homogeneidade da estrutura proporcionada pelas fibras, o painel MDF pode ser facilmente pintado e revestido, torneado, entalhado e perfurado. Além disso, por não possuir nós, veios e imperfeições típicas de uma madeira natural, o MDF tem a vantagem de poder ser usinado de diferentes formas. As opções de acabamento normalmente utilizadas são: "in natura", pintado e revestido com laminado de baixa pressão (BP) ou finish foil (FF) (ABIPA, 2008).

O MDF é amplamente empregado na indústria moveleira em frontais de portas, frentes de gaveta $\mathrm{e}$ outras peças mais elaboradas, com usinagens em bordas ou faces, como tampos de mesa, racks e estantes. $\mathrm{Na}$ construção civil, é utilizado como piso, rodapé, almofada de portas, batente, portas usinadas, peças torneadas (como balaústres de escadas e pés de mesas) e também em embalagens (ABIPA, 2008).

Tendo em vista as diferenças na matéria-prima utilizada e variações nos parâmetros do processo produtivo de painéis MDF entre as indústrias, a avaliação da qualidade de painéis com base nas propriedades 
físicas e mecânicas torna-se uma ferramenta importante por parte dos produtores para assegurar a confiabilidade desse produto no mercado consumidor, como as indústrias moveleiras e de construção civil.

Este trabalho teve como objetivo avaliar a qualidade de painéis MDF produzidos pelas indústrias brasileiras com base nos resultados de propriedades físicas e mecânicas dos painéis tipo "standard" com $15 \mathrm{~mm}$ de espessura.

\section{MATERIAL E MÉTODOS}

Foram selecionadas para esta pesquisa quatro empresas produtoras de painéis MDF, as quais foram identificadas pelas letras A, B, C e D. Para a avaliação das propriedades físicas e mecânicas dos painéis, foram coletados para todas as empresas, três painéis amostras do tipo standard, in natura, com espessura de $15 \mathrm{~mm}$ e densidade entre $0,65 \mathrm{~g} / \mathrm{cm}^{3}$ e $0,80 \mathrm{~g} / \mathrm{cm}^{3}$, produzidos com madeira de pinus. Para a empresa $\mathrm{D}$, além das três amostras de painéis de pinus foram coletadas também três amostras de painéis de eucalipto.

Após a amostragem e coleta dos painéis-teste, foram retirados os corpos de prova para ensaios físico-mecânicos. As dimensões e número de corpos de prova e as respectivas normas utilizadas estão apresentados na tabela 2 e ilustrados na figura 1.

Tabela 2. Corpos de prova para ensaios físico-mecânicos de painéis MDF.

Table 2. MDF samples for physical and mechanical testing.

\begin{tabular}{lcc}
\hline Ensaios - normas & $\begin{array}{c}\mathbf{N}^{\mathbf{0}} \text { de amostras } \\
\text { (cp/painel) }\end{array}$ & $\begin{array}{c}\text { Dimensões } \\
\text { (mm) }\end{array}$ \\
\hline Flexão Paralela (longitudinal) - EN 310 & 10 & $350 \times 50$ \\
Flexão Perpendicular (transversal) - EN 310 & 10 & $350 \times 50$ \\
Ligação Interna - EN 319 & 10 & $50 \times 50$ \\
Absorção de água / Inchamento em Espessura - EN 317 & 10 & $50 \times 50$ \\
Densidade - EN 323 & 10 & $50 \times 50$ \\
Teor de Umidade - EN 322 & 10 & $50 \times 50$ \\
Perfil de Densidade & 5 & $50 \times 50$ \\
\hline
\end{tabular}
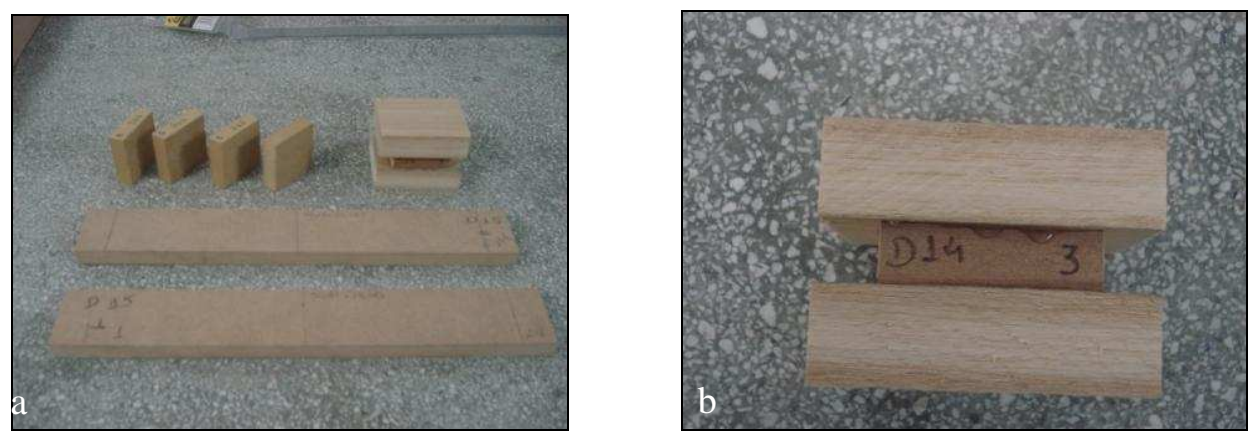

Figura 1. Corpos de prova para ensaios físico-mecânicos de painéis MDF. a) Corpos-de-prova de flexão (paralelo e perpendicular), densidade, perfil de densidade, absorção e inchamento, ligação interna; b) Corpos-de-prova de ligação interna colados aos suportes.

Figure 1. MDF Samples for physical and mechanical testing.

Os corpos de prova foram submetidos ao acondicionamento em câmara climática à temperatura de $20 \pm 2{ }^{\circ} \mathrm{C}$ e umidade relativa de $65 \pm 3 \%$, até atingirem a umidade de equilíbrio de aproximadamente $12 \%$.

$\mathrm{O}$ procedimento estatístico adotado foi o delineamento inteiramente casualizado, e os resultados dos ensaios foram analisados através de ANOVA e teste de Tukey ao nível de probabilidade de $95 \%$.

\section{RESULTADOS E DISCUSSÕES}

\section{Teor de umidade e densidade dos painéis}

Os valores médios de teor de umidade apresentados na tabela 3 indicam a variação entre os painéis na faixa de $8,94 \%$ a $10,20 \%$. Os painéis MDF de pinus da empresa $\mathrm{B}$ apresentaram teor de umidade inferior em relação aos painéis produzidos pelas demais empresas. Pode-se constatar também 
que, entre os painéis produzidos pela empresa $\mathrm{D}$, os de eucalipto apresentaram menor teor de umidade médio em relação aos painéis de pinus. Trata-se de uma diferença importante, tendo em vista que os dois tipos de painéis são produzidos pela mesma empresa e na mesma linha de produção. Essa diferença pode ser atribuída à maior eficiência da emulsão de parafina aplicada sobre as fibras de eucalipto durante o processo de desfibramento da madeira.

Tabela 3. Valores médios de teor de umidade e densidades dos painéis.

Table 3. Average values of moisture content and board densities.

\begin{tabular}{|c|c|c|}
\hline Empresa/Espécie & Teor de umidade $(\%)$ & Densidade $\left(\mathrm{g} / \mathrm{cm}^{3}\right)$ \\
\hline $\mathrm{A} / \mathrm{P}$ & 10,20 & $0,693 \mathrm{a}$ \\
\hline $\mathrm{C} / \mathrm{P}$ & 10,16 & $0,696 \mathrm{a}$ \\
\hline $\mathrm{D} / \mathrm{P}$ & 10,19 & $0,715 \mathrm{~b}$ \\
\hline $\mathrm{B} / \mathrm{P}$ & 8,99 & $0,736 \mathrm{c}$ \\
\hline $\mathrm{D} / \mathrm{E}$ & 8,94 & $0,756 \mathrm{~d}$ \\
\hline
\end{tabular}

Médias seguidas de mesma letra não diferem estatisticamente entre si ao nível de probabilidade de $95 \%$.

As variações nos valores médios de densidade dos painéis foram de $0,693 \mathrm{~g} / \mathrm{cm}^{3}$ a $0,756 \mathrm{~g} / \mathrm{cm}^{3}$ entre os produtos das diferentes empresas. Os painéis MDF das empresas A e $\mathrm{C}$ apresentaram médias estatisticamente inferiores em relação aos painéis das empresas B e D. Já os painéis MDF produzidos com madeira de eucalipto apresentaram valor médio de densidade estatisticamente superior em comparação aos painéis de pinus. A maior densidade média encontrada para os painéis de eucalipto pode ser atribuída à definição dos parâmetros do processo produtivo da empresa em relação a essa espécie, tendo em vista as características morfológicas das fibras de eucalipto, que apresentam menor comprimento e parede celular mais espessa.

\section{Perfil de densidade dos painéis}

Na tabela 4 estão apresentadas as variações encontradas no perfil de densidade a partir da face superior à face inferior do painel.

Tabela 4. Valores médios do perfil de densidade dos painéis.

Table 4. Average values of board density profile.

\begin{tabular}{lcccc}
\hline Empresa/Espécie & $\begin{array}{c}\text { Densidade máxima } \\
\text { superior }\left(\mathbf{g} / \mathbf{c m}^{\mathbf{3}}\right)\end{array}$ & $\begin{array}{c}\text { Densidade } \\
\text { mínima }\left(\mathbf{g} / \mathbf{c m}^{\mathbf{3}}\right)\end{array}$ & $\begin{array}{c}\text { Densidade máxima } \\
\text { inferior }\left(\mathbf{g} / \mathbf{c m}^{\mathbf{3}}\right)\end{array}$ & $\begin{array}{c}\text { Densidade média } \\
\left(\mathbf{g} / \mathbf{c m}^{\mathbf{3}}\right)\end{array}$ \\
\hline $\mathrm{A} / \mathrm{P}$ & 1,006 & 0,612 & 0,984 & 0,689 \\
$\mathrm{~B} / \mathrm{P}$ & 0,977 & 0,667 & 0,961 & 0,884 \\
$\mathrm{C} / \mathrm{P}$ & 1,015 & 0,611 & 1,006 & 0,688 \\
$\mathrm{D} / \mathrm{P}$ & 1,011 & 0,654 & 1,005 & 0,707 \\
$\mathrm{D} / \mathrm{E}$ & 0,984 & 0,650 & 0,966 & 0,747 \\
\hline
\end{tabular}

Os resultados obtidos indicam uma grande variação na densidade das faces superior e inferior em relação ao miolo do painel. Segundo Moslemi (1974), essa variação ocorre em decorrência do tempo de fechamento da prensa e diferenças na transferência de temperatura das camadas externas para a camada interna do painel durante o estágio de compressão das partículas do colchão até atingir a espessura final.

Para os painéis produzidos com madeira de eucalipto (D/E), pode-se constatar que os resultados foram diferenciados em relação aos painéis de pinus $(\mathrm{D} / \mathrm{P})$ no que se refere à sua maior densidade média $\mathrm{e}$ menores valores de densidades máxima inferior e superior. Esses valores indicam que houve, para os painéis de eucalipto, distribuição mais homogênea do perfil de densidade.

\footnotetext{
Absorção de água e inchamento em espessura

Os resultados de absorção de água após 2 e 24 horas de imersão em água apresentados na tabela 5 mostram uma variação de 2,06\% a 4,33\% para 2 horas de imersão e de 8,54\% a 16,38\% para 24 horas de imersão. Os resultados da análise estatística apontam diferenças significativas entre os painéis de pinus produzidos pelas quatro empresas e entre os painéis de pinus e de eucalipto. Os painéis produzidos com madeira de eucalipto apresentaram médias superiores e estatisticamente diferentes em comparação aos painéis produzidos com madeira de pinus, tanto para 2 quanto para 24 horas de imersão.

Os resultados de inchamento em espessura apresentados na tabela 5 indicam média estatisticamente inferior para os painéis de pinus produzidos pela empresa A em relação aos painéis das
} 
empresas B, C e D, e também quando comparados aos painéis de eucalipto. A relação direta entre a maior taxa de absorção de água e inchamento em espessura, como descrito por Maloney (1993) e Moslemi (1974), não foi confirmada para este lote de painéis MDF comerciais.

Cabe destacar que os valores médios de inchamento em espessura 24 horas, obtidos para este lote de painéis MDF comerciais, estão bem abaixo do requisito máximo de $12 \%$ estabelecido pela norma europeia EN 622-5:2006.

Tabela 5. Valores médios de absorção de água e inchamento em espessura.

Table 5. Average values of water absorption and thickness swelling.

\begin{tabular}{lcccc}
\hline Empresa/Espécie & $\mathbf{A A} \mathbf{- 2 h} \mathbf{( \% )}$ & $\mathbf{A A} \mathbf{- 2 4 h} \mathbf{( \% )}$ & IE - 2h (\%) & IE - 24h $\mathbf{( \% )}$ \\
\hline A/P & $2,06 \mathrm{a}$ & $8,54 \mathrm{a}$ & $0,84 \mathrm{a}$ & $4,01 \mathrm{a}$ \\
$\mathrm{B} / \mathrm{P}$ & $2,58 \mathrm{~b}$ & $11,08 \mathrm{~b}$ & $1,47 \mathrm{~b}$ & $7,53 \mathrm{c}$ \\
$\mathrm{C} / \mathrm{P}$ & $3,20 \mathrm{c}$ & $10,82 \mathrm{~b}$ & $1,73 \mathrm{~b}$ & $6,34 \mathrm{~b}$ \\
$\mathrm{D} / \mathrm{P}$ & $2,93 \mathrm{c}$ & $12,94 \mathrm{c}$ & $2,24 \mathrm{c}$ & $7,79 \mathrm{c}$ \\
$\mathrm{D} / \mathrm{E}$ & $4,33 \mathrm{~d}$ & $16,38 \mathrm{~d}$ & $2,41 \mathrm{c}$ & $7,72 \mathrm{c}$ \\
\hline
\end{tabular}

Médias seguidas de mesma letra não diferem estatisticamente entre si ao nível de probabilidade de $95 \%$. AA: absorção de água após 2 e 24 horas; IE: inchamento em espessura após 2 e 24 horas.

\section{Ligação interna}

Os resultados de ligação interna apresentados na tabela 6 indicam que há uma grande variação nos valores médios entre os painéis MDF produzidos pelas quatro empresas $(0,30$ a 0,53 MPa). Entre os painéis produzidos com madeira de pinus, o maior valor médio foi obtido para os painéis da empresa A, sendo esta a que alcançou valor mais próximo do requisito mínimo de 0,55 MPa estabelecido pela norma EN 622-5:2006. Os painéis produzidos com madeira de eucalipto foram as que apresentaram a menor média de ligação interna entre os painéis testados. Eleotério (2000) obteve para painéis MDF produzidos em escala laboratorial, com densidade de $0,700 \mathrm{~g} / \mathrm{cm}^{3}$ e $10 \%$ de resina ureia-formaldeído, valor de ligação interna de $0,29 \mathrm{MPa}$.

Tabela 6. Valores médios de densidade de painéis e ligação interna.

Table 6. Average values of board density and internal bond

\begin{tabular}{lcc}
\hline Empresa/Espécie & Densidade $\left(\mathbf{g} / \mathbf{c m}^{\mathbf{3}}\right)$ & Ligação interna $(\mathbf{M P a})$ \\
\hline $\mathrm{A} / \mathrm{P}$ & 0,693 & $0,53 \mathrm{~d}$ \\
$\mathrm{~B} / \mathrm{P}$ & 0,736 & $0,34 \mathrm{~b}$ \\
$\mathrm{C} / \mathrm{P}$ & 0,696 & $0,41 \mathrm{c}$ \\
$\mathrm{D} / \mathrm{P}$ & 0,715 & $0,35 \mathrm{~b}$ \\
$\mathrm{D} / \mathrm{E}$ & 0,756 & $0,30 \mathrm{a}$ \\
\hline
\end{tabular}

Médias seguidas de mesma letra não diferem estatisticamente entre si ao nível de probabilidade de $95 \%$.

Os resultados demonstraram também que não há uma relação direta entre a densidade dos painéis e valores de ligação interna. Segundo Moslemi (1974), a maior quantidade de material na formação do painel e o consequente aumento na sua densidade influencia de forma positiva na ligação interna.

\section{Flexão estática}

Os resultados de flexão estática apresentados na tabela 7 indicam variações de 2.886 a 3.241 MPa para MOE paralelo; 30,45 a 33,55 MPa para MOR paralelo; 3.004 a $3.312 \mathrm{MPa}$ para MOE perpendicular; e de 33,32 a 42,46 MPa para MOR perpendicular. Pode-se constatar que existem variações significativas entre os painéis produzidos pelas quatro empresas. Por outro lado, observa-se também que há variações significativas nos valores de densidade dos painéis, o que deve ter influenciado diretamente nos resultados de MOE e MOR em flexão estática.

Entre os resultados obtidos para painéis de diferentes empresas, cabe destacar que os resultados de MOR, tanto paralelo quanto perpendicular, obtidos para a empresa A, é muito superior em comparação aos resultados obtidos para as demais empresas, mesmo considerando que a densidade do painel é a 
menor entre todos os lotes de painéis testados. Os painéis produzidos com madeira de eucalipto apresentaram resultados superiores em relação aos painéis de pinus apenas para o MOE paralelo.

Tabela 7. Valores médios de densidade de painéis, MOE e MOR em flexão estática.

Table 7. Average values of board density, MOE and MOR in static bending.

\begin{tabular}{lcccccc}
\hline \multirow{2}{*}{ Empresa/Espécie } & \multicolumn{3}{c}{ Flexão Paralela } & \multicolumn{3}{c}{ Flexão Perpendicular } \\
\cline { 2 - 7 } & $\begin{array}{c}\text { Densidade } \\
\left(\mathbf{g} / \mathbf{c m}^{\mathbf{3}}\right)\end{array}$ & $\begin{array}{c}\text { MOE } \\
\mathbf{( M P a})\end{array}$ & $\begin{array}{c}\text { MOR } \\
\mathbf{( M P a})\end{array}$ & $\begin{array}{c}\text { Densidade } \\
\left(\mathbf{g} / \mathbf{c m}^{\mathbf{3}}\right)\end{array}$ & $\begin{array}{c}\text { MOE } \\
\mathbf{( M P a})\end{array}$ & $\begin{array}{c}\text { MOR } \\
\mathbf{( M P a})\end{array}$ \\
\hline $\mathrm{A} / \mathrm{P}$ & 0,686 & $3.046 \mathrm{c}$ & $39,10 \mathrm{~d}$ & 0,672 & $3.312 \mathrm{~d}$ & $42,46 \mathrm{c}$ \\
$\mathrm{B} / \mathrm{P}$ & 0,728 & $2.886 \mathrm{a}$ & $30,45 \mathrm{a}$ & 0,739 & $3.131 \mathrm{~b}$ & $33,32 \mathrm{a}$ \\
$\mathrm{C} / \mathrm{P}$ & 0,688 & $3.090 \mathrm{c}$ & $36,73 \mathrm{c}$ & 0,696 & $3.220 \mathrm{c}$ & $38,36 \mathrm{~b}$ \\
$\mathrm{D} / \mathrm{P}$ & 0,705 & $2.941 \mathrm{~b}$ & $32,78 \mathrm{~b}$ & 0,715 & $3.004 \mathrm{a}$ & $34,62 \mathrm{a}$ \\
$\mathrm{D} / \mathrm{E}$ & 0,734 & $3.241 \mathrm{~d}$ & $33,55 \mathrm{~b}$ & 0,729 & $3.159 \mathrm{~b}$ & $33,95 \mathrm{a}$ \\
\hline
\end{tabular}

Médias seguidas de mesma letra não diferem estatisticamente entre si ao nível de probabilidade de 95\%. MOE: módulo de elasticidade; MOR: módulo de ruptura.

Todos os painéis testados apresentaram valores médios de MOE e MOR paralelo superiores em comparação ao requisito mínimo estabelecido pela norma EN 622-5:2006, de 20 MPa para o MOR e de 2.200 MPa para o MOE. Os resultados obtidos por Eleotério (2000) para painéis MDF produzidos em escala laboratorial, com densidade de $0,700 \mathrm{~g} / \mathrm{cm}^{3}$ e $10 \%$ de resina ureia-formaldeído, foram de 22,31 MPa para o MOR e 2.770 MPa para o MOE.

\section{CONCLUSÕES}

- As variações estatisticamente significativas encontradas nas propriedades físicas e mecânicas dos painéis MDF indicam haver diferenças na qualidade de painéis MDF produzidos pelas indústrias brasileiras. Essas diferenças podem ser atribuídas às variações nos parâmetros do processo produtivo entre as empresas. Entretanto, todos os painéis avaliados nesta pesquisa apresentaram valores de propriedades físicas e mecânicas superiores aos requisitos mínimos da norma EM 622-5:2006, com exceção da ligação interna.

- Os painéis produzidos com madeira de eucalipto apresentaram valores médios inferiores de ligação interna e superiores de absorção de água em relação aos obtidos com painéis de pinus. Essas variações demonstram que as características diferenciadas das fibras de eucalipto (folhosas) em relação às fibras de pinus (coníferas) podem influenciar no processo de formação de painéis MDF e nas suas propriedades físicas e mecânicas.

\section{REFERÊNCIAS}

ABIPA - ASSOCIAÇÃO BRASILEIRA DA INDÚSTRIA DE PAINÉIS DE MADEIRA. Disponível em: http://www.abipa.org.br. Acesso 06 outubro 2008.

BNDES. Painéis de madeira no Brasil: panorama e perspectivas. Brasília: Ministério da Agricultura, Pecuária e Abastecimento. Agronegócios, 2008. v. 6, 84 p.

ELEOTÉRIO, J. R. Propriedades físicas e mecânicas de painéis MDF de diferentes densidades e teores de resina. 2000. 121 p. Dissertação (Mestrado) - Universidade de São Paulo, Piracicaba, 2000.

EUROPEAN COMMITTEE FOR STANDARDIZATION. Norma EN. 1993.

European Standard EN 622 - Tableros de fibras - Especificaciones. Parte 5: Requisitos de los tableros de fibras fabricados por proceso seco (MDF). 2006.

IWAKIRI, S. Painéis de Madeira Reconstituída. Curitiba: FUPEF, 2005. 247 p.

MALONEY, T. M. Modern particleboard \& dry-process fiberboard manufacturing. San Francisco: Miller Freeman, 1993. 657 p.

MOSLEMI, A. A. Particleboard. vol. 1: Materials. London: Southern Illinois University Press, 1974. 244 p. 